\title{
COMPRESSIVE SENSING BASED ENERGY DETECTOR
}

\author{
Eva Lagunas*, Shree Krishna Sharma, Symeon Chatzinotas and Björn Ottersten \\ Interdisciplinary Centre for Security, Reliability and Trust (SnT), \\ University of Luxembourg \\ 4, rue Alphonse Weicker L-2721, Luxembourg \\ Email: \{eva.lagunas,shree.sharma,symeon.chatzinotas,bjorn.ottersten $\} @$ uni.lu
}

\begin{abstract}
While most of research in Compressive Sensing (CS) has been focused on reconstruction of the sparse signal from very fewer samples than the ones required by the Shannon-Nyquist sampling theorem, recently there has been a growing interest in performing signal processing directly in the measurement domain. This new area of research is known in the literature as Compressive Signal Processing (CSP). In this paper, we consider the detection problem using a reduced set of measurements focusing on the Energy Detector (ED), which is the optimal Neyman-Pearson (NP) detector for random signals in Gaussian noise. In particular, we provide simple closed form expressions for evaluating the detection performance of ED when considering compressive measurements. The resulting equations reflect the loss due to $\mathrm{CS}$ and allow to determine the minimum number of samples to achieve certain detection performance.
\end{abstract}

Index Terms - Compressive Signal Processing, Compressive Sensing, Energy Detection

\section{INTRODUCTION}

The concept of Compressive Sensing (CS) [1-3] has attracted considerable research interest over the past years in the signal processing community. CS is a new signal processing technique for sampling analog signals which seeks to minimize the collection of redundant data in the acquisition step. The essence of this reduction of data is based on the sparsity or the sparse representation present in many common signals. The traditional CS approach is to recover the exact signal from its compressive measurements. The recovery or reconstruction process usually involves some kind of optimization procedure $[4,5]$. However, some signal processing applications like signal estimation, detection and classification, in which the main task is to solve inference problems, do not require

This work was supported by the National Research Fund, Luxembourg, under CORE project SpEctrum Management and Interference mitiGation in cognitive raDio satellite networks - SeMIGod, and CORE project SATellite SEnsor NeTworks - SATSENT.

* Corresponding author. the complete signal recovery. This has motivated a growing interest in working directly in the compressed domain, which has obvious advantages in terms of complexity in comparison with the traditional CS approach since the reconstruction step is omitted. This new area of research is known in the literature as Compressive Signal Processing (CSP) [6,7].

In this paper, we focus on the compressive signal detection problem. Signal detection is a well-known problem in signal processing, which involves deciding whether a particular signal is present or not from the observed data. Compressive signal processing has been applied to the detection problem in $[6,8-10]$. The most relevant work is [6], where theoretical bounds and probability expressions are provided for the detection of sparse deterministic signals. Deterministic signals are also considered in [8], for which the matched filter technique is the optimal Neyman-Pearson (NP) detector [11]. In [9], the compressive sensing detection of stochastic signals in a noisy environment is considered. In particular, the optimal projection or sampling matrix is derived in such a way that the classification error is minimized. An Energy Detector (ED) applied directly to the compressed signal is proposed in [10]. However, [10] is specific to Ultra-WideBand (UWB) Pulse Position Modulation (PPM) signals and only provides the corresponding bit error probability expression.

In contrast to previous works, we focus on the compressive detection of stochastic signals. For this, we follow the same philosophy as in [6] and focus on the NP setup, in which the probability of detection is maximized for a given probability of false alarm. As shown in [11], the NP detector (also known as likelihood-ratio test) for stochastic signal detection in the presence of White Gaussian Noise (WGN) is the ED. Therefore, we derive the compressive test statistic for ED and we formulate the corresponding equations related to its detection performance. In this context, the work in [12] deserves special attention, since it deals with a similar problem for the case of the NP detector. However, in [12], the Chi-squared distribution of the decision statistic is used to derive the operating characteristics of the NP detector resulting in cumbersome equations. Here, we make use of the central limit theorem to simplify the expressions of the probability of detection and the probability of false alarm for the CS-based ED which are 
expressed by a single $\mathcal{Q}$-function. The resulting equations can be used to easily quantify the effect of the reduced number of samples and to determine the minimum number of samples needed to achieve certain detection performance.

The remainder of this paper is structured as follows. In Section 2, the conventional ED is reviewed. In Section 3, we provide background on CS and introduce the compressive ED formulation. Section 3 also includes a comparatives discussion of the results obtained for the conventional and the CS-based ED. Supporting results are provided in Section 4, and Section 5 states the conclusion.

\section{CONVENTIONAL ENERGY DETECTION}

Signal detection is a binary decision problem [11] that can be formulated as follows:

$$
\left\{\begin{array}{l}
\mathcal{H}_{0}: \mathbf{x}=\mathbf{w} \\
\mathcal{H}_{1}: \mathbf{x}=\mathbf{s}+\mathbf{w}
\end{array}\right.
$$

where $\mathbf{x} \in \mathbb{R}^{N}$ denotes the observed data, $\mathbf{s} \in \mathbb{R}^{N}$ denotes the signal to be detected and $\mathbf{w} \sim \mathcal{N}\left(\mathbf{0}, \sigma_{w}^{2} \mathbf{I}_{N}\right)$ is the Additive White Gaussian Noise (AWGN) independent of the signal $\mathbf{s}$. The signal $\mathbf{s}$ is assumed to be a Gaussian random process with zero mean and variance $\sigma_{s}^{2}$, i.e., $\mathbf{s} \sim \mathcal{N}\left(\mathbf{0}, \sigma_{s}^{2} \mathbf{I}_{N}\right)$.

The detection algorithm has to decide if the signal $\mathbf{s}$ is present $\left(\mathcal{H}_{1}\right)$ or not $\left(\mathcal{H}_{0}\right)$ from the observation $\mathbf{x}$. For this, a test statistic $T(\mathbf{x})$ is computed from the observed data and compared to a threshold $\eta$. Let $P\left(\mathcal{H}_{i} ; \mathcal{H}_{j}\right)$ indicate the probability of deciding $\mathcal{H}_{i}$ when $\mathcal{H}_{j}$ is true. The probability of the algorithm to correctly detecting $\mathbf{s}$ is defined as Probability of Detection and is given by the following expression:

$$
P_{D}=P\left(\mathcal{H}_{1} ; \mathcal{H}_{1}\right)=P\left(T(\mathbf{x})>\eta \mid \mathcal{H}_{1}\right)
$$

Similarly, the Probability of False Alarm, $P_{F A}$, is the probability to falsely declaring the presence of the signal to be detected, and is given by

$$
P_{F A}=P\left(\mathcal{H}_{1} ; \mathcal{H}_{0}\right)=P\left(T(\mathbf{x})>\eta \mid \mathcal{H}_{0}\right) .
$$

Clearly, $P_{D}$ and $P_{F A}$ depend on each other. Based on the NP theory, if $P_{F A}$ is restricted to a maximum value $\lambda$, then the test statistic that maximizes $P_{D}$ is the likelihood-ratio test of the form

$$
T(\mathbf{x})=\frac{f_{1}(\mathbf{x})}{f_{0}(\mathbf{x})} \underset{\mathcal{H}_{1}}{\stackrel{\mathcal{H}_{0}}{\lessgtr}} \eta
$$

where $f_{i}(\mathbf{x})$ denotes the Probability Density Function (PDF) of $\mathbf{x}$ under hypothesis $\mathcal{H}_{i}$. It is shown in [11] that the test statistic $T(\mathbf{x})$ obtained from (4) is proportional to $\|\mathbf{x}\|_{2}^{2}=$ $\mathbf{x}^{T} \mathbf{x}$, which is the energy of the observed signal.

\subsection{Distribution of the Test Statistic and Detection Per- formance}

The test statistic $T(\mathbf{x})$ is a sum of $N$ squares of independent Gaussian random variables. As a consequence, $T(\mathbf{x})$ follows a central Chi-square distribution under hypothesis $\mathcal{H}_{0}$ and non-central Chi-square distribution under hypothesis $\mathcal{H}_{1}$ [13]. Using the central limit theorem, for large $N$ we have the following [13, 14],

$$
\left\{\begin{array}{l}
\mathcal{H}_{0}: T(\mathbf{x}) \sim \mathcal{N}\left(N \sigma_{w}^{2}, 2 N \sigma_{w}^{4}\right) \\
\mathcal{H}_{1}: T(\mathbf{x}) \sim \mathcal{N}\left(N\left(\sigma_{w}^{2}+\sigma_{s}^{2}\right), 2 N\left(\sigma_{w}^{2}+\sigma_{s}^{2}\right)^{2}\right) .
\end{array}\right.
$$

Therefore, from (2), (3) and (5) we obtain the following expressions:

$$
\begin{array}{r}
P_{D}=\mathcal{Q}\left(\frac{\eta-N \sigma_{w}^{2}(1+\gamma)}{\sqrt{2 N} \sigma_{w}^{2}(1+\gamma)}\right), \\
P_{F A}=\mathcal{Q}\left(\frac{\eta-N \sigma_{w}^{2}}{\sqrt{2 N} \sigma_{w}^{2}}\right),
\end{array}
$$

where $\mathcal{Q}(\cdot)$ denotes the complementary distribution function of the standard Gaussian and $\gamma$ is the Signal-to-Noise Ratio (SNR) defined as $\frac{\sigma_{s}^{2}}{\sigma_{w}^{2}}$.

From (6), and setting the $P_{F A}=\lambda$, we can rewrite $P_{D}$ as

$$
P_{D}=\mathcal{Q}\left(\frac{1}{1+\gamma}\left[\mathcal{Q}^{-1}(\lambda)-\gamma \sqrt{\frac{N}{2}}\right]\right)
$$

Finally, the minimum number of samples required to guarantee a minimum $P_{D}=\beta$ is

$$
N_{\min }=\left\lceil 2\left[\frac{1}{\gamma}\left(\mathcal{Q}^{-1}(\lambda)-(1+\gamma) \mathcal{Q}^{-1}(\beta)\right)\right]^{2}\right\rceil .
$$

\section{PROPOSED COMPRESSIVE ENERGY DETECTION}

We now consider the problem of detecting the signal $\mathbf{s}$ from the observation of $\mathbf{y}=\mathbf{\Phi} \mathbf{x}$, where $\boldsymbol{\Phi} \in \mathbb{R}^{M \times N}$, being $M<$ $N$. This corresponds to the conventional CS notation for the acquisition of sparse signals. The ratio $\rho=M / N$ is known as the compressive ratio. The binary decision problem can be formulated as,

$$
\left\{\begin{array}{l}
\mathcal{H}_{0}: \mathbf{y}=\mathbf{\Phi} \mathbf{w} \\
\mathcal{H}_{1}: \mathbf{y}=\boldsymbol{\Phi}(\mathbf{s}+\mathbf{w})
\end{array}\right.
$$

The distribution of $\mathbf{y}$ in (9) follow a zero-mean Gaussian multivariate distribution with covariance matrix equal to $\sigma_{w}^{2} \boldsymbol{\Phi} \boldsymbol{\Phi}^{T}$ for $\mathcal{H}_{0}$ and $\left(\sigma_{s}^{2}+\sigma_{w}^{2}\right) \boldsymbol{\Phi} \boldsymbol{\Phi}^{T}$ for $\mathcal{H}_{1}$. Therefore, we have the following probability density functions for the hypotheses $\mathcal{H}_{0}$ 
and $\mathcal{H}_{1}$,

$$
\left\{\begin{array}{l}
\mathcal{H}_{0}: f_{0}(\mathbf{y})=\frac{\exp \left(-\frac{1}{2} \mathbf{y}^{T}\left(\sigma_{w}^{2} \mathbf{\Phi} \boldsymbol{\Phi}^{T}\right)^{-1} \mathbf{y}\right)}{\sqrt{\left|\sigma_{w}^{2} \boldsymbol{\Phi} \boldsymbol{\Phi}^{T}\right|(2 \pi)^{M}}} \\
\mathcal{H}_{1}: f_{1}(\mathbf{y})=\frac{\exp \left(-\frac{1}{2} \mathbf{y}^{T}\left(\left(\sigma_{s}^{2}+\sigma_{w}^{2}\right) \boldsymbol{\Phi} \boldsymbol{\Phi}^{T}\right)^{-1} \mathbf{y}\right)}{\sqrt{\left|\left(\sigma_{s}^{2}+\sigma_{w}^{2}\right) \boldsymbol{\Phi} \boldsymbol{\Phi}^{T}\right|(2 \pi)^{M}}} .
\end{array}\right.
$$

In this work, we focus on a particular structure for the measurement matrix $\boldsymbol{\Phi}$, which is detailed in the following assumptions.

Assumption 1 The measurement matrix $\boldsymbol{\Phi}$ satisfies $\boldsymbol{\Phi} \boldsymbol{\Phi}^{T}=$ $\frac{1}{\rho} \boldsymbol{I}_{M}$. In other words, the rows of the measurement matrix are orthogonal and its columns have unit norm.

Note that Assumption 1 is approximately satisfied by matrices $\boldsymbol{\Phi}$ whose entries are zero-mean i.i.d. random variables with variance $1 / M$, when $N \rightarrow \infty$. Assumption 1 is satisfied as well by the measurement matrices employed by the widely used multi-coset sampler $[15,16]$.

According to (10) and making use of Assumption 1, the corresponding NP decision statistic is given by,

$T(\mathbf{y})=\left[\frac{\sigma_{w}^{2}}{\sigma_{s}^{2}+\sigma_{w}^{2}}\right]^{M / 2} \exp \left(\left(\frac{\sigma_{s}^{2}}{2 \frac{1}{\rho} \sigma_{w}^{2}\left(\sigma_{s}^{2}+\sigma_{w}^{2}\right)}\right)\|\mathbf{y}\|_{2}^{2}\right)$.

Clearly, in (11) it is shown that the optimal detector is proportional to the energy of the compressed signal, i.e. $T(\mathbf{y})=$ $\|\mathbf{y}\|_{2}^{2}=\mathbf{y}^{T} \mathbf{y}$. Therefore, defining a new threshold $\eta^{\prime}$, we have $T(\mathbf{y}) \lessgtr_{\mathcal{H}_{1}}^{\mathcal{H}_{0}} \eta^{\prime}$.

\subsection{Distribution of the Test Statistic and Detection Per- formance}

For convenience let us rewrite the test statistic $T(\mathbf{y})$ as follows:

$$
T(\mathbf{y})=\sum_{m=0}^{M-1} y^{2}(m)=\sum_{m=0}^{M-1}\left[\sum_{n=0}^{N-1} \varphi(m, n) x(n)\right]^{2}
$$

where $y(m), x(n)$ and $\varphi(m, n)$ denote the entries of $\mathbf{y}, \mathbf{x}$ and $\boldsymbol{\Phi}$, respectively. It is clear from (12) that the distribution of $T(\mathbf{y})$ depends on the distribution of $\varphi(m, n)$. Here, we focus on measurement matrices $\boldsymbol{\Phi}$ whose entries are zero-mean i.i.d. random variables with variance $1 / M$.

As for the conventional energy detector, we can apply the central limit theorem and, for large $N$ we have,

$$
\left\{\begin{array}{l}
\mathcal{H}_{0}: T(\mathbf{y}) \sim \mathcal{N}\left(N \sigma_{w}^{2}, 2 N\left(\frac{2+N}{M}\right) \sigma_{w}^{4}\right), \\
\mathcal{H}_{1}: T(\mathbf{y}) \sim \mathcal{N}\left(N\left(\sigma_{w}^{2}+\sigma_{s}^{2}\right), 2 N\left(\frac{2+N}{M}\right)\left(\sigma_{w}^{2}+\sigma_{s}^{2}\right)^{2}\right) .
\end{array}\right.
$$

Assuming that $2 \ll N$, we can do the following approximation:

$$
\left\{\begin{array}{l}
\mathcal{H}_{0}: T(\mathbf{y}) \approx \mathcal{N}\left(N \sigma_{w}^{2}, 2 N\left(\frac{1}{\rho}+1\right) \sigma_{w}^{4}\right), \\
\mathcal{H}_{1}: T(\mathbf{y}) \approx \mathcal{N}\left(N\left(\sigma_{w}^{2}+\sigma_{s}^{2}\right), 2 N\left(\frac{1}{\rho}+1\right)\left(\sigma_{w}^{2}+\sigma_{s}^{2}\right)^{2}\right) .
\end{array}\right.
$$

It can be observed from (14) that the mean of $T(\mathbf{y})$ under hypothesis $\mathcal{H}_{0}$ and $\mathcal{H}_{1}$ is not affected by the compressive sampling. The effect of working with a reduced number of measurements is reflected in the variance, which increases by a factor of $\frac{1}{\rho}+1$ with respect to the variance obtained for the conventional case.

Therefore, using (2), (3) and (14) we obtain the following expressions:

$$
\begin{gathered}
P_{D}=\mathcal{Q}\left(\frac{\eta^{\prime}-N \sigma_{w}^{2}(1+\gamma)}{\sqrt{2 N\left(\frac{1}{\rho}+1\right)} \sigma_{w}^{2}(1+\gamma)}\right), \\
P_{F A}=\mathcal{Q}\left(\frac{\eta^{\prime}-N \sigma_{w}^{2}}{\sqrt{2 N\left(\frac{1}{\rho}+1\right)} \sigma_{w}^{2}}\right) .
\end{gathered}
$$

Setting the value of $P_{F A}=\lambda$, we obtain a certain value of $\eta^{\prime}$ from the $P_{F A}$ expression in (15), Substituting the value of $\eta^{\prime}$ in the $P_{D}$ expression in (15), we obtain

$$
P_{D}=\mathcal{Q}\left(\frac{1}{1+\gamma}\left[\mathcal{Q}^{-1}(\lambda)-\gamma \sqrt{\frac{N}{2\left(\frac{1}{\rho}+1\right)}}\right]\right) .
$$

\subsection{Comparative Discussion}

Comparing (7) with (16), it is clear that the number of samples is an important parameter that determines the probability of detection. Due to the monotonically decreasing property of function $\mathcal{Q}(\cdot)$, it can be seen that the probability of detection of the conventional detector is equal or higher than that of the compressive detector. In other words, $P_{D}$ deteriorates for $\rho<1$, which is the case when compressive sampling is applied. Unfortunately, the $\mathcal{Q}$ function is not a linear decreasing function and the performance loss due to the reduced number of samples depends on the SNR.

From (8), it is confirmed that the SNR is also another key parameter in determining the probability of detection. Energy detector requires more samples at very low SNR values [17]. Essentially, some values of probability of false alarm and probability of detection can only be achieved at very low SNR when a large number of samples is captured. The consequences of this drawback are even worse in the CS setting, where the number of measurements is reduced.

\section{NUMERICAL RESULTS}

The goal of this section is to illustrate the differences between the conventional ED and the CS-based ED in different CS settings with the help of numerical results. For the measurement matrix $\boldsymbol{\Phi}$, we considered a matrix whose entries are zeromean i.i.d. gaussian random variables with variance $1 / M$. Therefore, Assumption 1 holds true and the equations derived in Section (3.1) apply. 


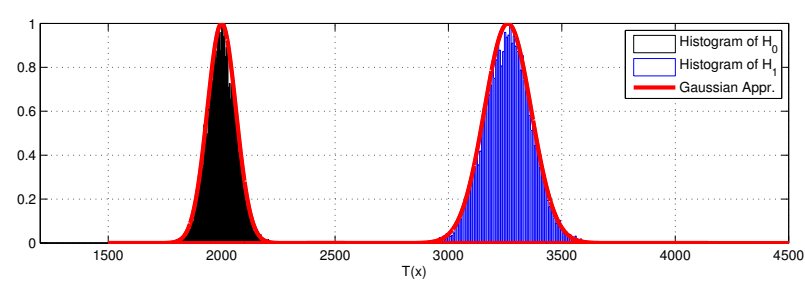

(a)

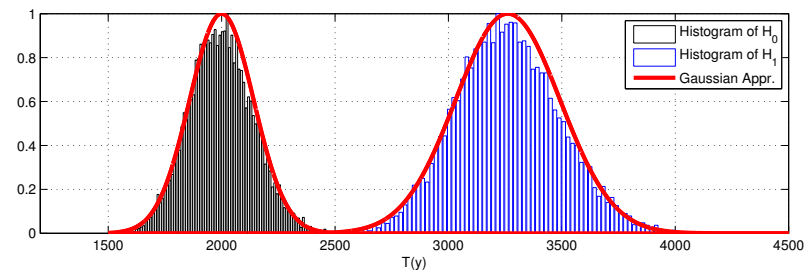

(b)

Fig. 1. Theoretical and empirical distributions of (a) $T(\mathbf{x})$ (conventional ED), and (b) $T(\mathbf{y})$ with $\rho=0.25$, for $\mathcal{H}_{0}$ and $\mathcal{H}_{1}, \gamma=-2 \mathrm{~dB}$ and $N=330$.

First of all, we show that central limit theorem provides good approximation for the distribution of $T(\mathbf{x})$ and $T(\mathbf{y})$. To this end, in Fig. 1(a) and Fig. 1(b), we depicted the histograms obtained from 10,000 Monte Carlo runs of $T(\mathbf{x})$ and $T(\mathbf{y})$, for both hypotheses $\mathcal{H}_{0}$ and $\mathcal{H}_{1}$, for a particular example with $\gamma=-2 \mathrm{~dB}$ and $\rho=0.25$ (for the compressive case). The number of full measurement samples is set to $N=2000$. In Fig. 1, it is observed that both distributions closely match the Gaussian approximations given in (5) and (13) (also shown in the figure with red solid lines). The effect of the compression is evident from Fig. 1, where the Gaussian curves depicted in Fig. 1(b) are wider bell-shaped compared to those in Fig. 1(a) due to the larger variance values.

Fig. 2 shows the $P_{D}$ versus $S N R$ results when $P_{F A}=$ $10^{-3}$ for different compression rates when $N=800$. As predicted from (7) and (16), the ED performance degrades as the number of measurements decreases. In particular, we can note from Fig. 2 that the conventional ED provides a $P_{D}=0.77$ for $\gamma=-7 \mathrm{~dB}$, while the CS-based ED strategies provide $P_{D}=\{0.62,0.41,0.18\}$ for $\rho=\{0.70,0.50,0.25\}$, respectively, for the same SNR value. The relation between $P_{D}, \rho$ and $\gamma$ is better illustrated in Fig. 3, where the value of $P_{D}$ is depicted for values of $\gamma$ ranging from $-5 \mathrm{~dB}$ to 5 $\mathrm{dB}$ and for different values of $\rho$. Once again, the increase in the detection probability is evident as we take more measurements. However, we again note that the relation between the compressive rate and $P_{D}$ can be significantly affected by the SNR.

In Fig. 4, we illustrate the receiver operating characteristic (ROC) curves, i.e., the relationship between $P_{F A}$ and $P_{D}$ for certain values of SNR. For each compressive ratio, we depict the ROC curves for $\gamma=\{-6,-8\} \mathrm{dB}$ and for two different

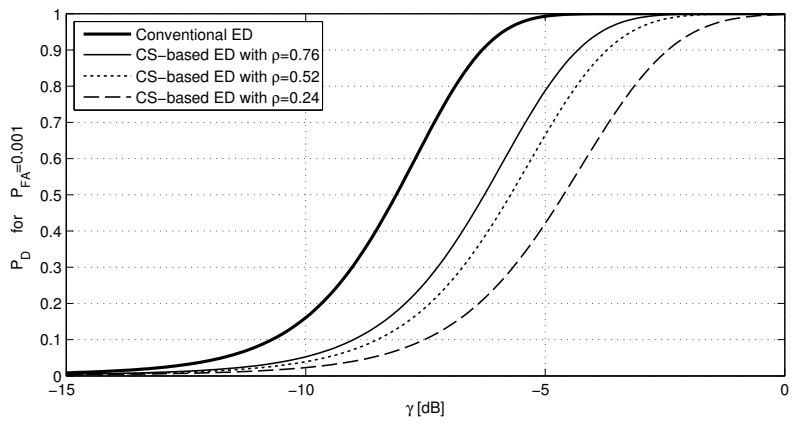

Fig. 2. $P_{D}$ versus $\gamma$ results when $P_{F A}=10^{-3}$ and $N=800$.

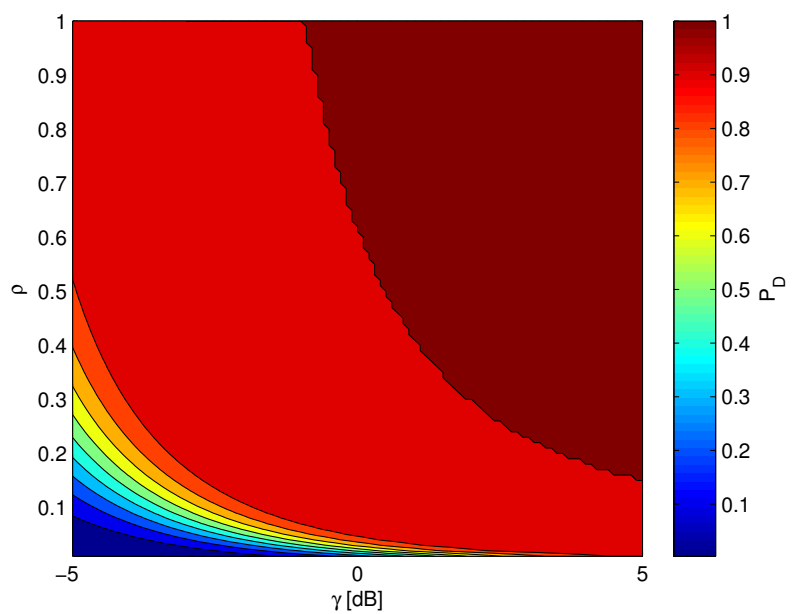

Fig. 3. Effect of $\gamma$ and $\rho$ in $P_{D}\left(P_{F A}=10^{-3}\right)$.

number of full measurement samples $N=\{500,100\}$. We show the theoretical ROC obtained from (15) together with the empirical ROC obtained from 10,000 Monte Carlo runs. It can be observed that the theoretical curves closely match the ones obtained with simulations. Note that the minimum number of samples required for perfect detection, i.e., $P_{D}=1$ and $P_{F A}=0$, when $\gamma=-6$ is around 3,600 samples according to (8). That explains why the ROC curves of Fig. 4(a) do not touch the upper-left corner of the figure. The curves in Fig. 4 show that the detector performance progressively deteriorates (i.e., approach the center of the figure) as $\rho$ and $\gamma$ decrease.

\section{CONCLUSION}

In this paper, we derived the NP detector for detecting a random signal in the presence of AWGN by making use of compressive measurements. We showed that the resulting detector is an ED and, subsequently, we formulated the equations describing its detection performance using the simple $\mathcal{Q}$-function. We focused on the widely-used measurement matrix whose entries are zero-mean i.i.d. random variables with variance $1 / M$ and we showed that the effect of the com- 


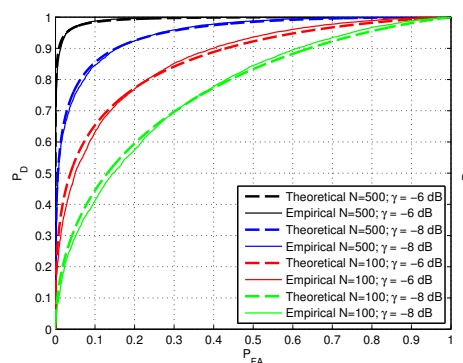

(a)

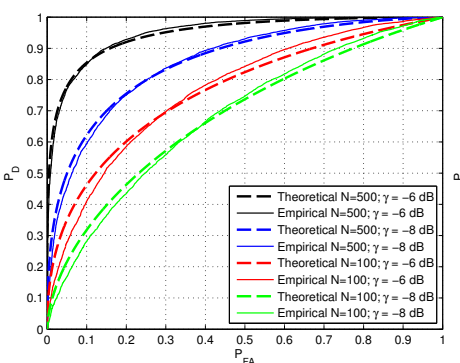

(b)

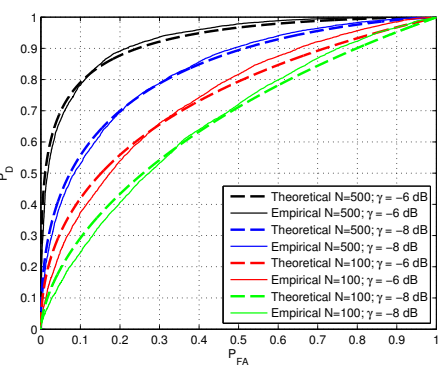

(c)

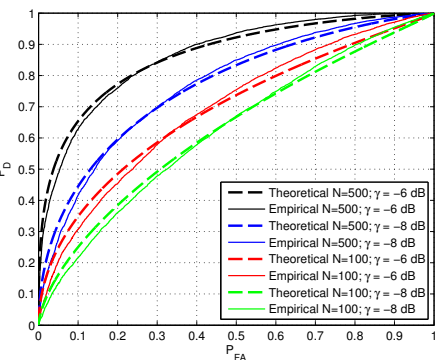

(d)

Fig. 4. ROC figures: (a) Conventional ED; (b) CS-based ED with $\rho=0.76$; (c) CS-based ED with $\rho=0.52$; (d) CS-based ED with $\rho=0.24$

pression is reflected on the variance of the test statistic's distribution, which is higher that that of the conventional ED. Numerical simulations support the theoretical results and confirm the loss due to the reduced number of samples.

\section{REFERENCES}

[1] E.J. Candes and M.B. Wakin, "An Introduction to Compressed Sampling," IEEE Signal Process. Mag., vol. 25, no. 2, pp. 21-30, Mar. 2008.

[2] D.L. Donoho, “Compressed Sensing," IEEE Trans. Inf. Theory, vol. 52, no. 4, pp. 1289-1306, Apr. 2006.

[3] S.K. Sharma, E. Lagunas, S. Chatzinotas, and B. Ottersten, "Application of Compressive Sensing in Cognitive Radio Communications: A Survey," IEEE Commun. Surveys and Tutorials, vol. PP, no. 99, pp. 1-24, Feb. 2016.

[4] J. A. Tropp and A. C. Gilbert, "Signal Recovery From Random Measurements Via Orthogonal Matching Pursuit," IEEE Trans. Inf. Theory, vol. 53, no. 12, pp. 4655-4666, Dec. 2007.

[5] E. J. Candes, J. Romberg, and T. Tao, "Stable Signal Recovery from Incomplete and Inaccurate Measurements," Comm. on Pure and Applied Mathematics, vol. 59, no. 8, pp. 1207-1223, Aug. 2006.

[6] M.A. Davenport, P.T. Boufounos, M.B. Wakin, and R.G. Baraniuk, "Signal Processing With Compressive Measurements," IEEE J. Sel. Topics Signal Process., vol. 4, no. 3, pp. 445-460, Apr. 2010.

[7] A. Jung, Z. Ben-Haim, F. Hlawatsch, and Y.C. Eldar, "Unbiased Estimation of a Sparse Vector in White Gaussian Noise," IEEE Trans. Inf. Theory, vol. 57, no. 12, pp. 7856-7876, Dec. 2011.

[8] J. Haupt and R. Nowak, "Compressive Sampling for Signal Detection," Int. Conf. on Acoustics, Speech and Signal Processing (ICASSP), Honolulu, HI, USA, Apr. 2007.
[9] J.E. Vila-Forcen, A. Artes-Rodriguez, and J. GarciaFrias, "Compressive Sensing Detection of Stochastic Signals," Annual Conf. in Information Sciences and Systems (CISS), Princeton, NJ, USA, Mar. 2008.

[10] S. Gishkori and G. Leus, "Compressive Sampling Based Energy Detection of Ultra-Wideband Pulse Position Modulation," IEEE Trans. Signal Process., vol. 61, no. 15, pp. 3866-3879, Aug. 2013.

[11] S.M. Kay, Fundamentals of Statistical Signal Processing, Volume II: Detection Theory, Prentice Hall, New Jersey, USA, 1998.

[12] B. Shankar, S. Chatterjee, and B. Ottersten, "Detection of Sparse Random Signals Using Compressive Measurements," Int. Conf. on Acoustics, Speech and Signal Processing (ICASSP), Kyoto, Japan, Mar. 2012.

[13] S. Atapattu, C. Tellambura, and H. Jiang, Energy Detection for Spectrum Sensing in Cognitive Radio, Springer, New York, USA, 2014.

[14] Y.C. Liang, Y. Zeng, E.C.Y. Peh, and A.T. Hoang, "Sensing-Throughput Tradeoff for Cognitive Radio Networks," IEEE Trans. Wireless Commun., vol. 7, no. 4, pp. 1326-1337, Apr. 2008.

[15] P. Feng, "Universal Minimum-Rate Sampling and Spectrum-Blind Reconstruction for Multiband Signals," PhD, University of Illinois at UrbanaChampaign, USA, 1997.

[16] R. Venkataramani and Y. Bresler, "Optimal SubNyquist Nonuniform Sampling and Reconstruction for Multiband Signals," IEEE Sig. Process. Letters, vol. 18, no. 8, pp. 443-446, Aug, 2011.

[17] D. Cabric and R.W. Tkachenko, A. Brodersen, "Experimental Study of Spectrum Sensing Based on Energy Detection and Network Cooperation," Int. Workshop on Technology and Policy for Accessing Spectrum (TAPAS), Boston, MA, Aug. 2006. 